PROCEEDINGS OF THE

AMERICAN MATHEMATICAL SOCIETY

Volume 131, Number 9, Pages 2963-2966

S 0002-9939(03)07094-1

Article electronically published on April 9, 2003

\title{
A NOTE ON THE IMBEDDING THEOREM OF BROWDER AND TON
}

\author{
J. BERKOVITS
}

(Communicated by N. Tomczak-Jaegermann)

\begin{abstract}
The imbedding theorem of Browder and Ton states that for any real separable Banach space $X$ there exist a real separable Hilbert space $H$ and a compact linear injection $\psi: H \rightarrow X$ such that $\psi(H)$ is dense in $X$. We shall give a short and elementary new proof to this result. We also briefly discuss the corresponding result without the completeness assumption.
\end{abstract}

\section{INTRODUCTION}

The imbedding theorem of Browder and Ton [7] can be viewed as an abstract version of classical imbedding theorems familiar in the context of function spaces. Indeed, let

$$
W^{m, p}(\Omega)=\left\{u \mid D^{\alpha} u \in L_{p}(\Omega) \quad \text { for all }|\alpha| \leq m\right\},
$$

where $\Omega$ is a bounded open set in $\mathbb{R}^{n}$ satisfying the uniform cone condition. If $1 \leq p<\infty, k-m \geq 1$ and

$$
\frac{1}{p}>\frac{1}{2}-\frac{k-m}{n},
$$

then by the Sobolev imbedding theorem (see [1, for instance) the natural injection $i: W^{k, 2}(\Omega) \rightarrow W^{m, p}(\Omega)$ is a compact linear map having a dense range in $W^{m, p}(\Omega)$.

In 1968 F. Browder and B.A. Ton proved the abstract version of the imbedding theorem. It states, on a purely abstract level, that for any real separable Banach space $X$ there exist a real separable Hilbert space $H$ and a compact linear injection $\psi: H \rightarrow X$ such that $\psi(H)$ is dense in $X$.

In their original paper, Browder and Ton used the imbedding theorem to obtain the so-called 'elliptic super-regularization' for operators from $X$ into the dual space $X^{*}$. Their approach is a generalization of the method of elliptic regularization used by Lions, Nirenberg and others (see the references given in [7]). The idea is to replace a given nonlinear elliptic equation by a mildly nonlinear elliptic equation of higher order, in which the nonlinear term is considered as a perturbation. A similar idea is later used for instance in [3], [2], 4], [5], [6], 8], [10] and [11.

The original proof of the imbedding theorem in [7] is quite lengthy. A shorter version based on the same reasoning can be found in 9 . We give a short and elementary new proof. Let $X$ be a real separable Banach space and $S=\left\{v_{1}, v_{2}, \ldots\right\}$ an infinite set of linearly independent vectors such that $\left\|v_{k}\right\|_{X}=1$ for all $k \in \mathbb{Z}_{+}$

Received by the editors May 30, 2002.

2000 Mathematics Subject Classification. Primary 47H05, 78M99.

Key words and phrases. Compact imbedding. 
and sp $S$ is dense in $X$. Taking into account a suitably restricted set of infinite linear combinations of vectors of $S$ we find a linear space $V$ such that $\operatorname{sp} S \subset V \subset X$ and $V$ can be naturally identified with a compact injective image of a closed subspace of $l^{2}$. Actually, we shall give a variant of the imbedding theorem without the completeness assumption. The imbedding theorem of Browder and Ton is then obtained as a corollary.

\section{The RESUlT}

Let $X$ be a real normed space and $\tilde{X}$ the essentially unique completion of $X$. The norm in $\tilde{X}$ is denoted by $\|\cdot\|_{\tilde{X}}$ and $\|x\|_{\tilde{X}}=\|x\|_{X}$ whenever $x \in X$.

Theorem 2.1. Let $X$ be a normed space and $S \subset X$ a countable subset. Then there exist a separable Hilbert space $H$ and a compact linear injection $\psi: H \rightarrow \tilde{X}$ such that $\operatorname{sp} S \subset \psi(H) \cap X$.

Proof. Without loss of generality we can assume that $S=\left\{v_{1}, v_{2}, \ldots\right\}$ is an infinite set of linearly independent vectors such that $\left\|v_{k}\right\|_{X}=1$ for all $k \in \mathbb{Z}_{+}$. Let $\left(a_{k}\right)_{k=1}^{\infty}$ be a real sequence such that $\left(a_{k}\right)_{k=1}^{\infty} \in l^{2}$. Then the series

$$
\sum_{k=1}^{\infty} \frac{a_{k}}{k} v_{k}
$$

converges in $\tilde{X}$. Indeed, denoting $s_{n}=\sum_{k=1}^{n} \frac{a_{k}}{k} v_{k}$ we have

$$
\left\|s_{n+p}-s_{n}\right\|_{X} \leq \sum_{k=n+1}^{n+p} \frac{\left|a_{k}\right|}{k} \leq \sqrt{\sum_{k=n+1}^{n+p} \frac{1}{k^{2}}} \sqrt{\sum_{k=n+1}^{n+p}\left|a_{k}\right|^{2}}
$$

for all $n \in \mathbb{Z}_{+}$and $p=1,2,3, \ldots$. Hence $\left(s_{n}\right)$ is a Cauchy sequence in $X$ and it converges in $\tilde{X}$. Note that the representation $u=\sum_{k=1}^{\infty} \frac{a_{k}}{k} v_{k},\left(a_{k}\right)_{k=1}^{\infty} \in l^{2}$, is not necessarily unique. Define the map $i: l^{2} \rightarrow \tilde{X}$ by setting

$$
i(\vec{a})=\sum_{k=1}^{\infty} \frac{a_{k}}{k} v_{k}
$$

for all $\vec{a}=\left(a_{k}\right)_{k=1}^{\infty} \in l^{2}$. Then it is easy to see that $i$ is linear and by the estimate (2.1) (with the usual convention that the sum over an empty set is zero)

$$
\|i(\vec{a})\|_{\tilde{X}} \leq c_{0}\|\vec{a}\|_{l^{2}}
$$

where $c_{0}=\sqrt{\sum_{k=1}^{\infty} \frac{1}{k^{2}}}$. Hence the mapping $i: l^{2} \rightarrow \tilde{X}$ is continuous. Moreover, the map $i$ is compact since it is a uniform limit of operators having finite dimensional range. Indeed, denoting $i_{n}(\vec{a})=\sum_{k=1}^{n} \frac{a_{k}}{k} v_{k}$ we get by (2.1)

$$
\left\|i(\vec{a})-i_{n}(\vec{a})\right\|_{\tilde{X}} \leq \sqrt{\sum_{k=n+1}^{\infty} \frac{1}{k^{2}}} \sqrt{\sum_{k=n+1}^{\infty}\left|a_{k}\right|^{2}} .
$$

Thus

$$
\left\|i-i_{n}\right\|=\sup _{\|\vec{a}\|=1}\left\|i(\vec{a})-i_{n}(\vec{a})\right\|_{\tilde{X}} \leq \sqrt{\sum_{k=n+1}^{\infty} \frac{1}{k^{2}}}
$$


proving the assertion. Denote $W_{0}=\operatorname{Ker}(i)$, which is a closed linear subspace of $l^{2}$. Define a real separable Hilbert space $H$ by setting

$$
H=W_{0}^{\perp}=\left\{\vec{a} \in l^{2} \mid \vec{a} \perp W_{0}\right\} .
$$

Then $H \cong l^{2} / W_{0}=l^{2} / \operatorname{Ker}(i)$ and consequently the map $\psi=\left.i\right|_{H}: H \rightarrow \tilde{X}$ is a linear compact injection. Moreover, denoting by $P: l^{2} \rightarrow H$ the orthonormal projection, we have $i\left(\vec{e}_{j}\right)=\psi\left(P \vec{e}_{j}\right)=v_{j} / j \in X$ for all $j \in \mathbb{Z}_{+}$, where $\vec{e}_{j}=\left(\delta_{j, k}\right)_{k=1}^{\infty}$. Clearly the subset $S_{H}:=\psi^{-1}(S)$ of $H$ is countable and $\psi\left(\operatorname{sp} S_{H}\right)=\operatorname{sp} S$. Hence $\operatorname{sp} S \subset \psi(H) \cap X$, completing the proof.

Corollary 2.2. Let $X$ be a real separable space. Then there exist a separable Hilbert space $H$ and a compact linear injection $\psi: H \rightarrow \tilde{X}$ such that $\psi(H) \cap X$ is dense in $X$.

Proof. Let $S=\left\{v_{1}, v_{2}, \ldots\right\}$ be an infinite set of linearly independent vectors such that $\left\|v_{k}\right\|=1$ for all $k \in \mathbb{Z}_{+}$and $\operatorname{sp} S$ is dense in $X$. Clearly $\operatorname{sp} S$ is also dense in $\tilde{X}$. Thus by Theorem 2.1 there exist a real separable Hilbert space $H$ and a linear compact injection $\psi: H \rightarrow \tilde{X}$ such that $\operatorname{sp} S \subset \psi(H) \cap X$, completing the proof.

Corollary 2.3 (Imbedding Theorem of Browder and Ton). Let $X$ be a real separable Banach space. Then there exist a separable Hilbert space $H$ and a compact linear injection $\psi: H \rightarrow X$ such that $\psi(H)$ is dense in $X$.

Proof. Now $X=\tilde{X}$ and the the conclusion follows from Corollary 2.2

We close this note with a few remarks, which may clarify the reasoning. Let $X$ be a real separable Banach space and let $S=\left\{v_{1}, v_{2}, \ldots\right\}$ be an infinite set of linearly independent vectors such that $\left\|v_{k}\right\|=1$ for all $k \in \mathbb{Z}_{+}$and $\operatorname{sp} S$ is dense in $X$. In view of the proofs above it is relevant to define a linear subspace of $X$ by setting

$$
V=\left\{u \in X \mid u=\sum_{k=1}^{\infty} \frac{a_{k}}{k} v_{k},\left(a_{k}\right)_{k=1}^{\infty} \in l^{2}\right\} .
$$

Clearly sp $S \subset V \subset X$ and hence $V$ is dense in $X$. Identifying any pair of sequences $\left(a_{k}\right)_{k=1}^{\infty} \in l^{2}$ and $\left(b_{k}\right)_{k=1}^{\infty} \in l^{2}$ such that $\sum_{k=1}^{\infty} \frac{a_{k}}{k} v_{k}=\sum_{k=1}^{\infty} \frac{b_{k}}{k} v_{k}$ in $X$, gives the quotient space identified with a closed subspace $H$ of $l^{2}$ needed in Corollary 2.3.

\section{REFERENCES}

[1] R.A. Adams, Sobolev Spaces, Academic Press, 1975. MR 56:9247

[2] Y.I. Alber, The solution of nonlinear equations with monotone operators in a Banach space, Siberian Math. J. 16 (1) (1975) pp. 1-8. MR 51:6512

[3] H. Amann, An existence and unicity theorem for the Hammerstein equation in Banach spaces, Math. Z. 111 (3) (1969) pp. 175-190. MR 40:7894

[4] J. Berkovits, On the degree theory for nonlinear mappings of monotone type, Ann. Acad. Sci. Fenn. Ser. A1, Dissertationes, 58 (1986). MR 87f:47084

[5] J. Berkovits and V. Mustonen, On the topological degree for mappings of monotone type, Nonlinear Anal., TMA, 10 (1986) pp. 1373-1383. MR 88b:47073

[6] J. Berkovits and M. Tienari, Topological degree for some classes of multis with applications to hyperbolic and elliptic problems involving dicontinuous nonlinearities, Dynamic Systems and Applications 5 (1996) pp. 1-18. MR 96m:47112

[7] F.E. Browder and B.A. Ton, Nonlinear functional equations in Banach spaces and elliptic super-regularization, Math. Z. 105 (1968) pp. 177-195. MR 38:582 
[8] A.A. Khan, A regularization approach for variational inequalities, Comput. Math. Appl. 42 (1-2), (2001) pp. 65-74. MR 2002b:49020

[9] D. Pascali and S. Sburlan, Nonlinear Mappings of Monotone Type, Editura Academiei, 1978. MR 80g:47056

[10] C.G. Simader, Weak solutions of the Dirichlet problem for strongly nonlinear elliptic differential equations, Math. Z. 150 (1) (1976) pp. 1-26. MR 54:8018

[11] J.R.L. Webb, On the Dirichlet problem for strongly nonlinear elliptic operators in unbounded domains, J. Lond. Math. Soc. 10 (1975) pp. 163-170. MR 52:14644

Department of Mathematical Sciences, University of Oulu, P.O. Box 3000, Fin-90014 Oulu, Finland

E-mail address: juha.berkovits@oulu.fi 